\title{
Quantitatively differentiating microstructures of tissues by frequency distributions of Mueller matrix images
}

Chao $\mathrm{He}$

Honghui $\mathrm{He}$

Xianpeng Li

Jintao Chang

Ye Wang

Shaoxiong Liu

Nan Zeng

Yonghong $\mathrm{He}$

Hui Ma 


\title{
Quantitatively differentiating microstructures of tissues by frequency distributions of Mueller matrix images
}

\author{
Chao He, ${ }^{a, b}$ Honghui He, ${ }^{a}$ Xianpeng Li, ${ }^{\text {a,c }}$ Jintao Chang, ${ }^{\text {a,c }}$ Ye Wang, ${ }^{\text {a,c }}$ Shaoxiong Liu, ${ }^{d}$ Nan Zeng, ${ }^{a}$ \\ Yonghong $\mathrm{He}^{\mathrm{a}}$ and $\mathrm{Hui} \mathrm{Ma}^{\mathrm{a}, \mathrm{c}, \star}$ \\ ${ }^{a}$ Tsinghua University, Institute of Optical Imaging and Sensing, Graduate School at Shenzhen, Shenzhen Key Laboratory for Minimal Invasive \\ Medical Technologies, Shenzhen 518055, China \\ ${ }^{\mathrm{b} T}$ Tsinghua University, Department of Biomedical Engineering, Beijing 100084, China \\ ${ }^{\mathrm{C} T}$ Tsinghua University, Department of Physics, Beijing 100084, China \\ 'Shenzhen Sixth People's Hospital (Nanshan Hospital) Huazhong University of Science and Technology Union Shenzhen Hospital, \\ Shenzhen 518052, China
}

\begin{abstract}
We present a new way to extract characteristic features of the Mueller matrix images based on their frequency distributions and the central moments. We take the backscattering Mueller matrices of tissues with distinctive microstructures, and then analyze the frequency distribution histograms (FDHs) of all the matrix elements. For anisotropic skeletal muscle and isotropic liver tissues, we find that the shapes of the FDHs and their central moment parameters, i.e., variance, skewness, and kurtosis, are not sensitive to the sample orientation. Comparisons among different tissues further indicate that the frequency distributions of Mueller matrix elements and their corresponding central moments can be used as indicators for the characteristic microstructural features of tissues. A preliminary application to human cervical cancerous tissues shows that the distribution curves and central moment parameters may have the potential to give quantitative criteria for cancerous tissues detections. (C) The Authors. Published by SPIE under a Creative Commons Attribution 3.0 Unported License. Distribution or reproduction of this work in whole or in part requires full attribution of the original publication, including its DOI. [DOI: 10.1117/1.JBO.20.10.105009]
\end{abstract}

Keywords: Mueller matrix; frequency distribution histogram; central moment; tissue; microstructures.

Paper 150437R received Jun. 29, 2015; accepted for publication Sep. 23, 2015; published online Oct. $21,2015$.

\section{Introduction}

Polarization imaging can provide rich microstructural and optical information of tissues for diagnostic purposes. ${ }^{1-5}$ Since a Mueller matrix provides the most comprehensive characterization of the polarization features, ${ }^{6}$ it has been applied to differentiate various abnormal tissues, such as skin cancer, ${ }^{7}$ cervical cancer, ${ }^{8-10}$ colon cancer, ${ }^{11-13}$ liver fibrosis,,${ }^{14}$ and so on. ${ }^{15-18}$ For anisotropic tissues, previous studies have shown that the Mueller matrix elements may change significantly with the orientation of the sample, making quantitative characterization of the microstructural features very difficult. ${ }^{19-20}$ It was also pointed out that the structural information encoded in a Mueller matrix can be presented by other transformed parameters with more explicit physics meanings. ${ }^{10}$ In fact, the abundant information carried by the Mueller matrix may allow us to identify the characteristic features of abnormal tissues without using high-resolution images. The two-dimensional (2-D) images of Mueller matrix elements can be reduced into a group of quantitative or semiquantitative, orientation insensitive parameters that reveal clearly the key structural features of the samples.

In this paper, we present a new way based on the statistical method to transform the 2-D images of the Mueller matrix elements into frequency distribution histogram (FDH), and central moment parameters. We study the quantitative influence of sample orientation on the frequency distributions of Mueller

*Address all correspondence to: Hui Ma, E-mail: mahui@tsinghua.edu.cn matrix elements, and then analyze the relations between the microstructures of tissues and the shapes of the FDHs. The experimental results show that the central moment analysis can provide us a group of orientation insensitive parameters representing the dominant features of tissues. The preliminary results of human cervical cancerous tissues show that the analysis method presented in this paper may serve as quantitative or semiquantitative criteria for cancerous tissue detection.

\section{Methods and Materials}

\subsection{Experimental Setup}

We adopted the backscattering Mueller matrix measurement configuration based on the dual rotating retarder method. ${ }^{21-23}$ As shown in Figs. 1(a) and 1(b), the illuminating light from the light-emitting diode (Source, $633 \mathrm{~nm}, 1 \mathrm{~W}$ ) passes through the lens (L1, Thorlabs), and the polarization states generator consisting of a polarizer (P1, Thorlabs) and a quarter-wave plate (R1, Thorlabs). The photons backscattered from the sample pass through the polarization states analyzer (PSA) consisting of the analyzing quarter-wave plate (R2, Thorlabs) and polarizer ( $\mathrm{P} 2$, Thorlabs), then are recorded by a charge-coupled device camera (QImaging 32-0122A, 12 bit, Canada) after passing through another lens (L2, Thorlabs). There is an oblique incident angle $(\theta=20 \mathrm{deg})$ between the illumination light and the detection direction to avoid the surface reflection of the sample. During the Mueller matrix measurements, the sample can be rotated in the imaging $(\mathrm{X}-\mathrm{Y})$ plane. As shown in 


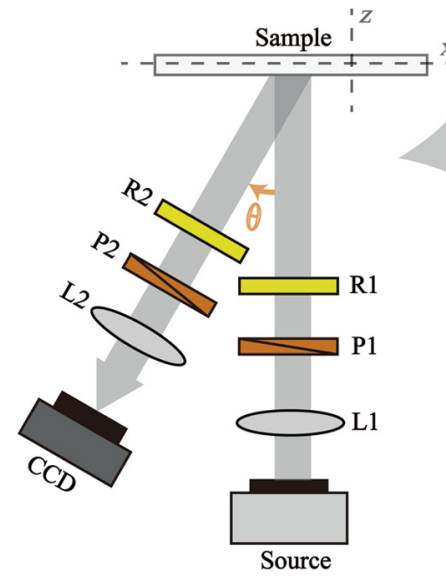

(a)

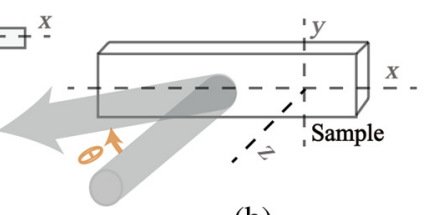

(b)

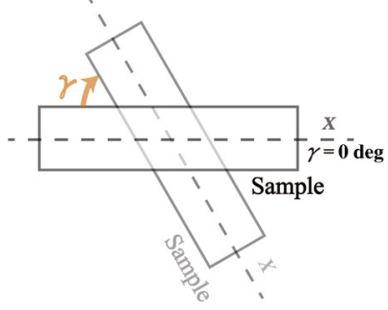

(c)
Fig. 1 (a) Schematic of experimental setup for the backscattering Mueller matrix measurement. P1, P2: polarizer; R1, R2: quarterwave plate; L1, L2: lens. The oblique incident angle $\theta$ is about $20 \mathrm{deg}$ to avoid the surface reflection from the sample. The diameter of the illumination area is about $1.5 \mathrm{~cm}$. (b) three-dimensional sketch of the sample. (c) During the measurements, the angle $\gamma$ can be varied from 0 deg to 180 deg.

Fig. 1(c), we can vary the angle $\gamma$ from 0 deg to 180 deg while keeping the center of the sample unchanged.

For the experimental setup shown in Fig. 1, the polarizers $\mathrm{P} 1$ and $\mathrm{P} 2$ are fixed in the horizontal direction, while the wave plates R1 and R2 are rotated with a fixed rate $\theta_{1}=5 \theta_{2}$, where $\theta_{1}$ and $\theta_{2}$ are the rotation angles of the wave plates $\mathrm{R} 1$ and $\mathrm{R} 2$, respectively. The Fourier series intensities can be given by Eq. (1),

$I=\alpha_{0}+\sum_{n=1}^{12}\left(\alpha_{n} \cos 2 \mathrm{n} \theta_{1}+\beta_{n} \sin 2 \mathrm{n} \theta_{1}\right)$

where $\alpha_{n}$ and $\beta_{n}$ are the Fourier coefficients. Using $\alpha_{n}$ and $\beta_{n}$, we can calculate the Mueller matrix elements according to Ref. 21. In order to record robust images, we measure the Mueller matrices of standard samples such as air and wave plate in the transmission mode and then calibrate the imaging system using the method proposed by Chenuault et al. ${ }^{24}$ The Mueller matrix elemental accuracy is tested by measuring the high extinction polarizers. After the calibration in transmission direction, the PSA arm of the system is rotated to the backscattered direction as shown in Fig. 1. We also measure the backscattering Mueller matrices of some samples with known polarization properties such as the microsphere solutions. In this work, the mean measurement errors of the diagonal and nondiagonal elements are less than $0.5 \%$ and $0.3 \%$, respectively. It is shown in our analysis of experimental results that the elemental uncertainty of the Mueller matrix measurement does not change the main characteristic features of the FDH, as well as the central moment parameters.

\subsection{Biological Tissue Samples}

In previous studies, we have taken the 2-D backscattering Mueller matrix images of different tissues, and analyzed the relations between their characteristic microstructural features

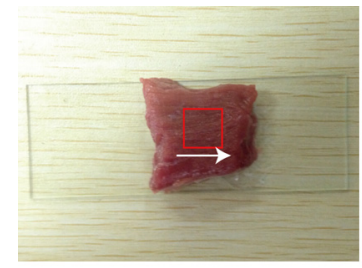

(a)

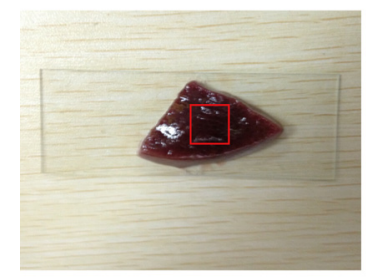

(c)

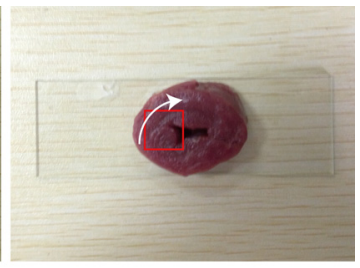

(b)

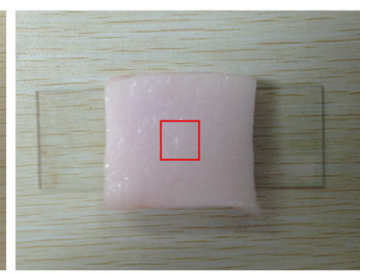

(d)
Fig. 2 Photographs of biological samples: (a) bovine skeletal muscle tissue, (b) chicken heart muscle tissue, (c) porcine liver tissue, and (d) porcine fat tissue. The areas marked by the red squares show the $1 \mathrm{~cm} \times 1 \mathrm{~cm}$ imaging regions. For the bovine skeletal and chicken heart muscle samples, the white arrow lines indicate the orientations of the muscle fibers. During the Mueller matrix imaging process, the surface of the sample was kept flat.

and the Mueller matrix elements. ${ }^{10}$ The tissue samples are shown in Fig. 2: (a) bovine skeletal muscle, (b) chicken heart muscle, (c) porcine liver, and (d) porcine fat. Both the bovine skeletal muscle and the chicken heart muscle are anisotropic, but the fibers are mostly aligned in the same direction for the bovine muscle sample while aligned concentrically around the ventricle for the chicken heart sample. ${ }^{25}$ Porcine liver and fat tissues are close to isotropic, but the liver sample contains many hexagonal boundaries of hepatic lobules, which are connective tissues of birefringence. ${ }^{10}$ The use of the animal tissues in this study was approved by the Administrative Committee on Animal Research of the Graduate School at Shenzhen, Tsinghua University. From the backscattering Mueller matrix images shown in Fig. 3, we can obtain abundant structural information of these samples. For instance, the anisotropy of tissues may originate from both the optical birefringence and cylindrical scatterers, which can be distinguished by the features in different Mueller matrix elements. ${ }^{10}$ The contributions due to the fibrous scatterers are encoded in the $\mathrm{m} 12, \mathrm{~m} 21, \mathrm{~m} 13$, and $\mathrm{m} 31$ elements $(\mathrm{m} 12=\mathrm{m} 21, \mathrm{~m} 13=\mathrm{m} 31)$, while those due to the birefringence are encoded in the $\mathrm{m} 24, \mathrm{~m} 42, \mathrm{~m} 34$, and $\mathrm{m} 43$ elements $(\mathrm{m} 24=-\mathrm{m} 42, \mathrm{~m} 34=-\mathrm{m} 43)$. The orientation of fibrous structures and the depolarization power can also be extracted from the Mueller matrix elements. ${ }^{10}$

Since the Mueller matrix contains abundant information on the tissue samples, we may not have to rely on the 2-D images to identify their characteristic features. It can be helpful to find a method to transform the 2-D images of Mueller matrix elements into a group of quantitative or semiquantitative, orientation insensitive parameters, which are crucial for the extraction of the dominant microstructural information of samples.

\subsection{Central Moment Analysis}

To quantitatively evaluate the Mueller matrix elements, we adopt the central moment method for statistical analysis of frequency distributions. ${ }^{26}$ 


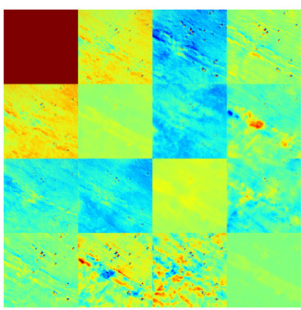

(a)

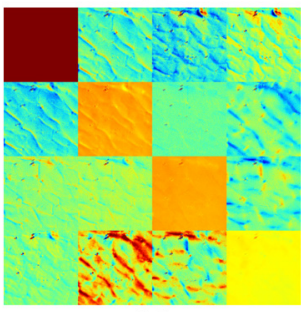

(c)

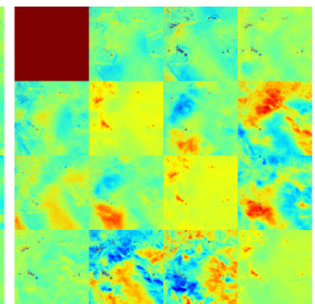

(b)

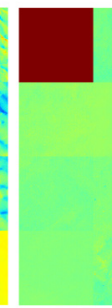

(d)

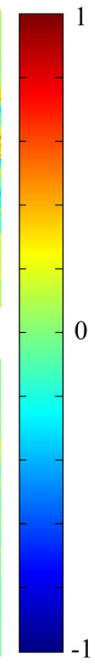

Fig. 3 Two-dimensional (2-D) images of backscattering Mueller matrices of biological samples: (a) bovine skeletal muscle, (b) chicken heart muscle, (c) porcine liver, and (d) porcine fat. The Mueller matrix elements are all normalized by the $\mathrm{m} 11$. The color bar is from -1 to 1 for $\mathrm{m} 11, \mathrm{~m} 22, \mathrm{~m} 33$, and $\mathrm{m} 44$, and from -0.1 to 0.1 for other elements. The imaging areas are indicated by the red squares in Fig. 2. For the skeletal muscle sample, the orientation angle of the fibers is about 30 deg.

$$
\begin{aligned}
\mu & =P 1=E(X), \\
\sigma^{2} & =P 2=\operatorname{Var}(X), \\
\text { skewness } & =P 3=\frac{E(X-\mu)^{3}}{\sigma^{3}}, \\
\text { kurtosis } & =P 4=\frac{E(X-\mu)^{4}}{\sigma^{4}} .
\end{aligned}
$$

Suppose we have a random variable $X$, whose central moments: expected value, variance, skewness, and kurtosis are exactly represented by Eq. (2). ${ }^{27}$ Here, the expected value $\mathrm{P} 1$ is the mean value of an FDH. The second central moment $\mathrm{P} 2$ is called the variance usually denoted by $\sigma^{2}$, where $\sigma$ represents the standard deviation of the FDH. A small P2 indicates that the measured data tend to be distributed close to the expected value, while a large $\mathrm{P} 2$ indicates that the data points are spread out around the expected value and from each other. The third and fourth central moments $\mathrm{P} 3$ and $\mathrm{P} 4$ represent the skewness and kurtosis of the FDH, respectively. P3 (skewness) shows the asymmetry of the FDH. The skewness value can be positive or negative. A negative (or positive) skewness value indicates that the tail on the left side (or the right side) of the FDH is longer or fatter than the right side (or the left side). P4 (kurtosis) indicates the "peakedness" of the FDH. It is a descriptor of the shape of a probability distribution. The detailed physical interpretations of kurtosis, skewness, and the central moments can be found in Refs. 26 and 28. In this paper, we first record the 2-D backscattering Mueller matrix images of samples, and then transfer the pixel images to FDHs by statistical analysis. Finally, the central moment method is applied to the FDHs of Mueller matrix elements.

\section{Results and Discussion}

\subsection{Characteristic Features in Mueller Matrix Elements of Tissues at Different Orientations}

Figure 4 shows the FDH of the backscattering Mueller matrix elements of the bovine skeletal muscle tissue. Previous studies have shown that the anisotropic muscle fibers can seriously affect the polarization measurement, resulting in difficulties of the structural information extraction. ${ }^{19}$ In this study, we rotate the skeletal muscle sample, measure the Mueller matrix images in different orientations, and then choose a square area of $700 \times$ 700 pixels at the same location of the sample. In Fig. 4, the horizontal axis of each FDH represents the value of the pixel from the corresponding Mueller matrix element, while the vertical axis represents the distributing probability. There are four experimental curves of the bovine skeletal muscle sample along $30 \mathrm{deg}$ (black lines), $60 \mathrm{deg}$ (red lines), $120 \mathrm{deg}$ (green lines), and $150 \mathrm{deg}$ (blue lines) directions [the angle is indicated as $\gamma$ in Fig. 1(c)]. To make the evaluation quantitative, we apply the central moment method to the FDHs, and calculate the parameters $\mathrm{P} 1, \mathrm{P} 2, \mathrm{P} 3$, and $\mathrm{P} 4$, which are listed in Table 1. Figure 4 and Table 1 show that the FDHs are transformed into the quantitative central moment parameters: the expected value $\mathrm{P} 1$, variance $\mathrm{P} 2$, skewness $\mathrm{P} 3$, and kurtosis $\mathrm{P} 4$ which all together characterize the position and shape of the FDH curves.

It can be observed from Fig. 4 and Table 1 that, as the orientation direction of the fibrous sample changed, most FDHs of the elements move except the m11, m14, m41, and m44, showing the influence of sample orientation on Mueller matrix measurements. Compared with the 2-D images, the FDHs and central moment parameters still reveal clearly and quantitatively the same main structural features of tissues as summarized in our previous studies: ${ }^{10,18}(1)$ the Mueller matrix shown in Fig. 4 and Table 1 is nondiagonal, the $\mathrm{m} 22$ and $\mathrm{m} 33$ elements are not equal (for instance, for the muscle sample along $30 \mathrm{deg}$ the P1 of $\mathrm{m} 22$ and $\mathrm{m} 33$ are 0.114 and 0.241 , respectively), and testifying that the bovine skeletal muscle is anisotropic. (2) We also notice that the FDHs of the $\mathrm{m} 24, \mathrm{~m} 34, \mathrm{~m} 42$, and $\mathrm{m} 43$ elements represent slight variations (the absolute value of $\mathrm{P} 1$ varies from 0.001 to 0.022 in different angles), indicating the existence of birefringence in this sample. Further analysis of the positive or negative values of these elements can provide the orientation axis information. ${ }^{18}$ (3) The values of the diagonal elements $\mathrm{m} 22, \mathrm{~m} 33$, and $\mathrm{m} 44$ are relatively small (P1 mostly distributed in 0.02 to $0.3)$, indicating a large depolarization property. ${ }^{10}$

The influences of sample orientation on the Mueller matrix elements are serious. Mueller matrix images of an anisotropic tissue sample along different directions may look like from different samples. However, Fig. 4 shows that the FDH curves can help us to distinguish that it is a different sample or just the same sample along a different orientation; as the sample rotated, the positions of the curves move but their shapes almost remain the same. Table 1 quantitatively confirms that when we rotate the muscle sample, the values of P1 change periodically, which indicates the variations of the positions for the FDHs. Meanwhile, the values of P2 (variance), P3 (skewness), and P4 (kurtosis) display very small changes, indicating the similar shapes (distributing width, asymmetry, and peakedness) of the FDHs. It should be pointed out that, compared to $\mathrm{P} 2$ and $\mathrm{P} 4$, the variation of $\mathrm{P} 3$ seems to be prominent in Table 1. It is because the length of the confidence interval of $\mathrm{P} 3$ is related to the absolute value of the skewness. When the skewness is very small, the confidence 

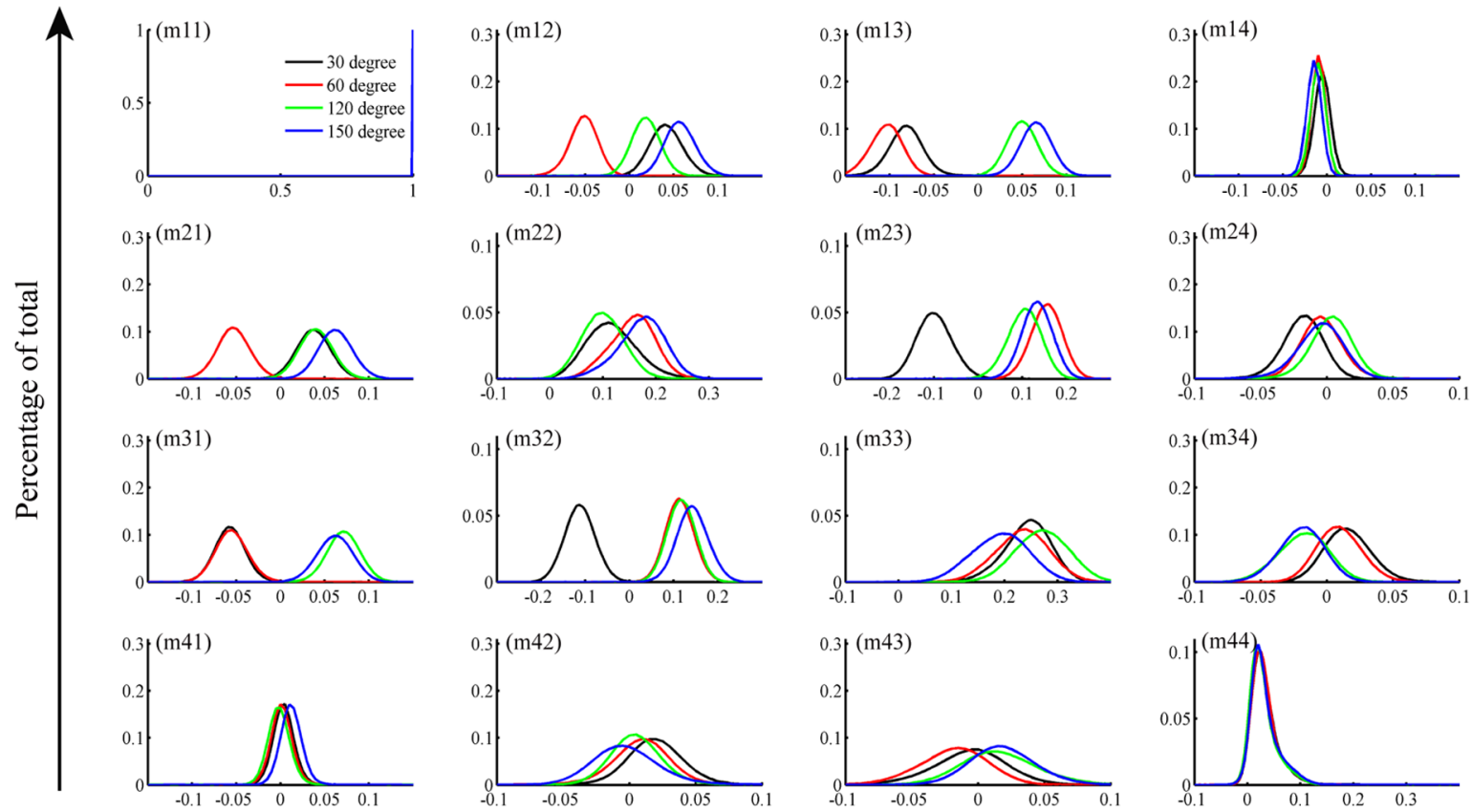

Values of Mueller matrix elements

Fig. 4 Frequency distribution histogram (FDH) of Mueller matrix elements of bovine skeletal muscle tissue along different orientation directions: 30 deg (black lines), 60 deg (red lines), 120 deg (green lines), and 150 deg (blue lines). The areas under the curves are normalized to 1 , and the horizontal axis is divided into 400 parts.

Table 1 Central moment parameters of the Mueller matrix elements for bovine skeletal muscle tissue.

\begin{tabular}{|c|c|c|c|c|c|c|c|c|c|c|c|c|c|c|c|}
\hline & $\mathrm{m} 12$ & $\mathrm{~m} 13$ & m14 & $\mathrm{m} 21$ & 22 & m23 & m24 & ר1 & m32 & m33 & m34 & $\mathrm{m} 41$ & m42 & m43 & m44 \\
\hline $30 \mathrm{deg} / \mathrm{P} 1$ & 0.039 & 0.084 & 8 & 0.035 & 0.114 & -0.102 & .021 & 0 & -0.115 & .241 & 0.013 & 0.001 & .017 & 0.009 & 0.029 \\
\hline $60 \mathrm{deg} / \mathrm{P} 1$ & 4 & 107 & 012 & - & 4 & 0.152 & 007 & 58 & 2 & 1 & 7 & & .006 & 21 & 0.029 \\
\hline $120 \mathrm{deg} / \mathrm{P} 1$ & 0.016 & 0.047 & .012 & 0.037 & 0.097 & 0.102 & 0.001 & 0.069 & 0.115 & 268 & & -0 & .003 & 0.013 & .026 \\
\hline $150 \mathrm{deg}$ & 0.055 & 0.063 & 0.017 & 0.060 & 0.171 & 0.131 & 7 & 0.059 & 0.140 & .189 & -0.022 & 0.009 & $-c$ & 0.016 & 0.029 \\
\hline 30 de & 0.016 & . & .008 & 0.017 & $0.04<$ & 0.035 & 0.013 & 0.015 & 0.030 & .039 & 0.015 & 0.010 & 0.018 & 0.025 & 0.021 \\
\hline $60 \mathrm{de}$ & 0.014 & 0.0 & 0.007 & 0.016 & 0.037 & 0.031 & 0.014 & 0.016 & 0.028 & 0.0 & 0.015 & 0.010 & 0.019 & 0.023 & 0.021 \\
\hline 2 & 0.014 & 0 & $r$ & 0.016 & 0.035 & 0.0 & 0.0 & 0.0 & 0.0 & 4 & 0.017 & 0. & 0.017 & 0.025 & 0.022 \\
\hline $150 \mathrm{deg} / \mathrm{P} 2$ & 0.015 & 0.015 & 0.007 & 0.017 & 0.039 & 0.030 & 0.015 & 0.018 & 0.031 & .047 & 0.015 & 0.010 & 0.022 & 0.022 & 0.023 \\
\hline $30 \mathrm{deg} / \mathrm{P} 3$ & 0.071 & -0.016 & -0.016 & -0.002 & 0.230 & 0.103 & 0.141 & 0.050 & 0.037 & 15 & 0.141 & 0.064 & 0.055 & -0.178 & 0.824 \\
\hline $60 \mathrm{de}$ & -0.121 & -0.107 & -0.048 & 0.029 & -0.176 & -0.063 & 0.110 & 0.065 & 0.052 & -0.075 & 0.114 & 85 & -0.189 & -0.171 & 0.820 \\
\hline $120 d$ & 0.028 & -0.002 & -0.043 & 0.012 & 0.070 & -0.108 & -0.106 & -0.017 & 0.002 & -0.029 & -0.124 & 0.087 & 0.151 & 0.156 & 0.887 \\
\hline $150 \mathrm{deg} / \mathrm{P} 3$ & 0.106 & -0.017 & -0.028 & 0.038 & -0.253 & -0.020 & -0.194 & -0.045 & 0.045 & -0.078 & -0.112 & 0.078 & 0.249 & 0.132 & 0.931 \\
\hline 30 & 2.372 & $2.50 \varsigma$ & 2.445 & 2.359 & 2.446 & 2.357 & 2.444 & 2.426 & 2.367 & 2.470 & 2.386 & 2.4 & 2.492 & 2.527 & 3.388 \\
\hline 60 & 2.440 & 82 & 2.372 & 2.393 & 2.376 & 2.3 & 2.472 & 2.393 & 2.365 & 2.360 & 58 & 2.487 & 2.609 & 2.420 & 3.362 \\
\hline $120 \mathrm{deg} / \mathrm{P} 4$ & 2.350 & 2.361 & 2.384 & 2.344 & 2.341 & 2.350 & 2.453 & 2.362 & 2.325 & 2.312 & 2.336 & 2.444 & 2.521 & 2.439 & 3.388 \\
\hline $150 \mathrm{deg} / \mathrm{P} 4$ & 2.372 & 2.364 & 377 & 2.356 & 2.470 & 2.335 & 2.493 & 2.340 & 2.387 & 2.295 & 2.368 & 2.447 & 2.589 & 2.432 & 3.397 \\
\hline
\end{tabular}


interval becomes wider. ${ }^{27}$ Therefore, according to the small values of $\mathrm{P} 3$ in this work, its confidence interval is about -0.3 to 0.3 . The values of $\mathrm{P} 3$ shown in Table 1 mean that the asymmetry of the FDH curves shown in Fig. 4 can be treated as almost the same.

For comparisons, we also take the backscattering Mueller matrix images of porcine liver tissue along different sample orientations. The liver tissues are primarily isotropic, but contain many thin hexagonal structures around the isotropic liver tissues, which are identified as birefringent connective tissues. ${ }^{10}$ Figure 5 shows the FDHs of the liver sample at 30 deg (black lines), $60 \mathrm{deg}$ (red lines), $120 \mathrm{deg}$ (green lines), and $150 \mathrm{deg}$ (blue lines) directions, respectively. The central moment parameters P1, P2, P3, and P4 are listed in Table 2.

It can be observed from Fig. 5 and Table 2 that, the FDHs of porcine liver tissues have isotropic structural features, which are different from those of the fibrous skeletal muscle shown in Fig. 4 and Table 1: (1) the Mueller matrix shown in Fig. 5 and Table 2 is nearly diagonal (the P1 values of the $\mathrm{m} 12$, $\mathrm{m} 21, \mathrm{~m} 13$, and $\mathrm{m} 31$ are close to 0 ), and the $\mathrm{m} 22$ and $\mathrm{m} 33$ elements are equal, representing isotropic dominant properties. (2) The $\mathrm{m} 24, \mathrm{~m} 34, \mathrm{~m} 42$, and $\mathrm{m} 43$ elements show slight differences (almost $\mathrm{m} 24=-\mathrm{m} 42, \mathrm{~m} 34=-\mathrm{m} 43$ ), indicating the existence of birefringent connective tissues. (3) Compared to the skeletal muscle sample, the values of the diagonal elements of the liver tissue are larger (P1 mostly distributed in 0.25 to 0.5$)$, reminding a smaller depolarization property. We also notice that when the sample rotates, the FDHs of the Mueller matrix elements all keep the same except the m24, m34, $\mathrm{m} 42$, and $\mathrm{m} 43$, confirming that for isotropic samples, the influence of orientation on polarization measurements is limited. Again, the FDHs and central moment parameters reveal more clearly and quantitatively the characteristic features of Mueller matrix elements for isotropic samples. ${ }^{10}$

From the data shown in Tables 1 and 2, we can conclude that: for the same sample placed along different orientations, the values of P2, P3, and P4 for the Mueller matrix elements almost do not change, while the value of P1 can be varied (for the anisotropic sample) or constant (for the isotropic sample). The central moment analysis of the FDHs provides us a tool to transform the complicated 2-D Mueller matrix images to a group of quantitative indicators of dominant structural properties of tissues. More importantly, using the $\mathrm{P} 2, \mathrm{P} 3$, and $\mathrm{P} 4$, we can obtain the main intrinsic properties of samples without the influence from orientation variations.

\subsection{Comparisons of Tissue Samples with Distinctive Microstructures}

In Sec. 3.1, we have found that the shapes of the FDHs of Mueller matrix elements are orientation insensitive, therefore may be used as indicators for intrinsic microstructural features of different tissues. To study the relationship between the FDHs and the structural properties, we take the Mueller matrices of the tissue samples as shown in Fig. 2. Figure 6 represents the experimental results of bovine skeletal muscle tissue (black lines), porcine liver tissue (red lines), chicken heart tissue (green lines), and porcine fat tissue (blue lines), the imaging area is a square of $700 \times 700$ pixels. During the measurements, the orientations of the samples are kept the same, for the bovine skeletal muscle sample its fibers are along the $30 \mathrm{deg}$ direction $(\gamma=30 \mathrm{deg})$.

We can see from Fig. 6 that the FDHs of different tissues have very different distributions. The corresponding central
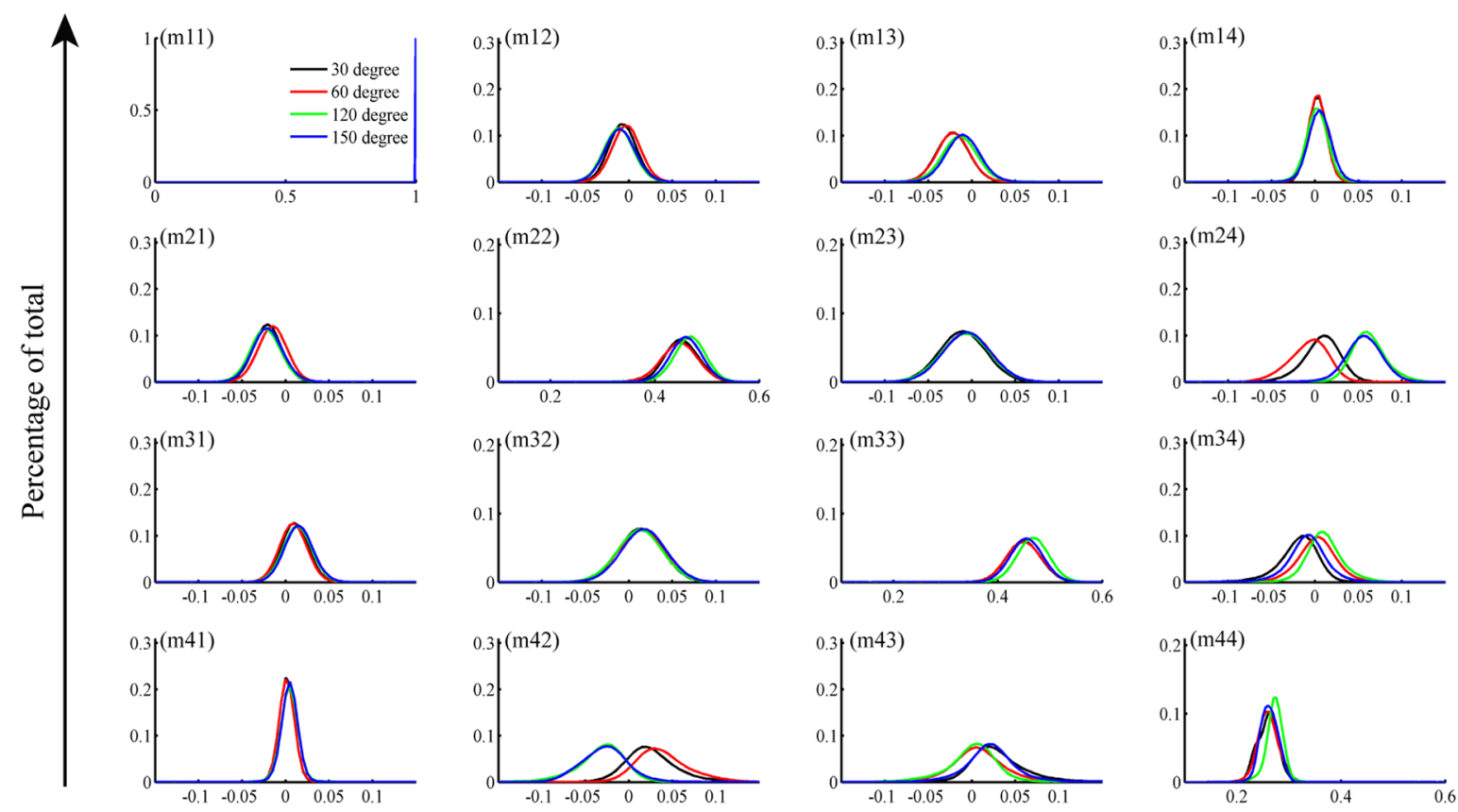

Values of Mueller matrix elements

Fig. 5 Frequency distribution histogram (FDH) of Mueller matrix elements of porcine liver tissue along different orientation directions: $30 \mathrm{deg}$ (black lines), $60 \mathrm{deg}$ (red lines), $120 \mathrm{deg}$ (green lines), and $150 \mathrm{deg}$ (blue lines). The areas under the curves are normalized to 1 , and the horizontal axis is divided into 400 parts. 
Table 2 Central moment parameters of the Mueller matrix elements for porcine liver tissue.

\begin{tabular}{|c|c|c|c|c|c|c|c|c|c|c|c|c|c|c|c|}
\hline & $\mathrm{m} 12$ & $\mathrm{~m} 13$ & $\mathrm{~m} 14$ & $\mathrm{~m} 21$ & $\mathrm{~m} 22$ & m23 & m24 & m31 & m32 & m33 & m34 & $\mathrm{m} 41$ & $\mathrm{~m} 42$ & $\mathrm{~m} 43$ & $\mathrm{~m} 44$ \\
\hline $30 \mathrm{deg} / \mathrm{P} 1$ & -0.010 & -0.024 & 0.000 & -0.023 & 0.449 & -0.013 & 0.005 & 0.008 & 0.010 & 0.447 & -0.021 & -0.001 & 0.022 & 0.027 & 0.257 \\
\hline $60 \mathrm{deg} / \mathrm{P} 1$ & -0.006 & -0.024 & 0.000 & -0.018 & 0.444 & -0.008 & -0.010 & 0.006 & 0.011 & 0.448 & -0.001 & -0.001 & 0.036 & 0.005 & 0.257 \\
\hline $120 \mathrm{deg} / \mathrm{P} 1$ & -0.014 & -0.016 & 0.000 & -0.025 & 0.464 & -0.010 & 0.059 & 0.011 & 0.010 & 0.465 & 0.009 & 0.002 & -0.034 & -0.002 & 0.271 \\
\hline $150 \mathrm{deg} / \mathrm{P} 1$ & -0.013 & -0.013 & 0.003 & -0.023 & 0.457 & -0.008 & 0.054 & 0.012 & 0.015 & 0.452 & -0.012 & 0.002 & -0.030 & 0.019 & 0.260 \\
\hline $30 \mathrm{deg} / \mathrm{P} 2$ & 0.014 & 0.017 & 0.009 & 0.014 & 0.028 & 0.024 & 0.018 & 0.014 & 0.022 & 0.029 & 0.019 & 0.008 & 0.025 & 0.027 & 0.017 \\
\hline $60 \mathrm{deg} / \mathrm{P} 2$ & 0.014 & 0.017 & 0.009 & 0.014 & 0.031 & 0.024 & 0.020 & 0.014 & 0.023 & 0.029 & 0.019 & 0.008 & 0.027 & 0.026 & 0.017 \\
\hline $120 \mathrm{deg} / \mathrm{P} 2$ & 0.015 & 0.018 & 0.011 & 0.015 & 0.027 & 0.025 & 0.017 & 0.014 & 0.023 & 0.027 & 0.018 & 0.009 & 0.024 & 0.025 & 0.014 \\
\hline $150 \mathrm{deg} / \mathrm{P} 2$ & 0.015 & 0.017 & 0.011 & 0.015 & 0.027 & 0.024 & 0.018 & 0.014 & 0.023 & 0.027 & 0.019 & 0.008 & 0.024 & 0.025 & 0.015 \\
\hline $30 \mathrm{deg} / \mathrm{P} 3$ & 0.040 & -0.004 & 0.030 & 0.003 & 0.073 & 0.004 & -0.250 & 0.006 & -0.020 & 0.028 & -0.447 & 0.000 & 0.360 & 0.496 & -0.101 \\
\hline $60 \mathrm{deg} / \mathrm{P} 3$ & -0.010 & 0.009 & -0.047 & -0.026 & -0.064 & -0.003 & -0.299 & 0.016 & -0.024 & 0.053 & -0.090 & 0.009 & 0.436 & 0.143 & 0.077 \\
\hline $120 \mathrm{deg} / \mathrm{P} 3$ & 0.021 & 0.027 & -0.016 & 0.041 & -0.095 & -0.010 & 0.283 & 0.019 & -0.010 & 0.000 & 0.286 & -0.009 & -0.465 & -0.417 & -0.220 \\
\hline $150 \mathrm{deg} / \mathrm{P} 3$ & 0.039 & -0.015 & 0.007 & 0.058 & -0.017 & 0.008 & -0.080 & 0.034 & -0.009 & 0.003 & -0.172 & -0.047 & 0.055 & 0.131 & 0.094 \\
\hline $30 \mathrm{deg} / \mathrm{P} 4$ & 2.374 & 2.408 & 2.437 & 2.369 & 2.364 & 2.357 & 2.537 & 2.362 & 2.373 & 2.376 & 2.804 & 2.400 & 2.696 & 3.040 & 2.312 \\
\hline $60 \mathrm{deg} / \mathrm{P} 4$ & 2.374 & 2.410 & 2.492 & 2.370 & 2.415 & 2.351 & 2.440 & 2.359 & 2.369 & 2.334 & 2.566 & 2.416 & 2.670 & 2.713 & 2.417 \\
\hline $120 \mathrm{deg} / \mathrm{P} 4$ & 2.397 & 2.512 & 2.575 & 2.390 & 2.465 & 2.369 & 2.578 & 2.376 & 2.391 & 2.370 & 2.724 & 2.536 & 2.841 & 2.970 & 2.761 \\
\hline $150 \mathrm{deg} / \mathrm{P} 4$ & 2.414 & 2.439 & 2.488 & 2.400 & 2.404 & 2.368 & 2.476 & 2.396 & 2.381 & 2.377 & 2.836 & 2.493 & 2.547 & 3.071 & 2.257 \\
\hline
\end{tabular}
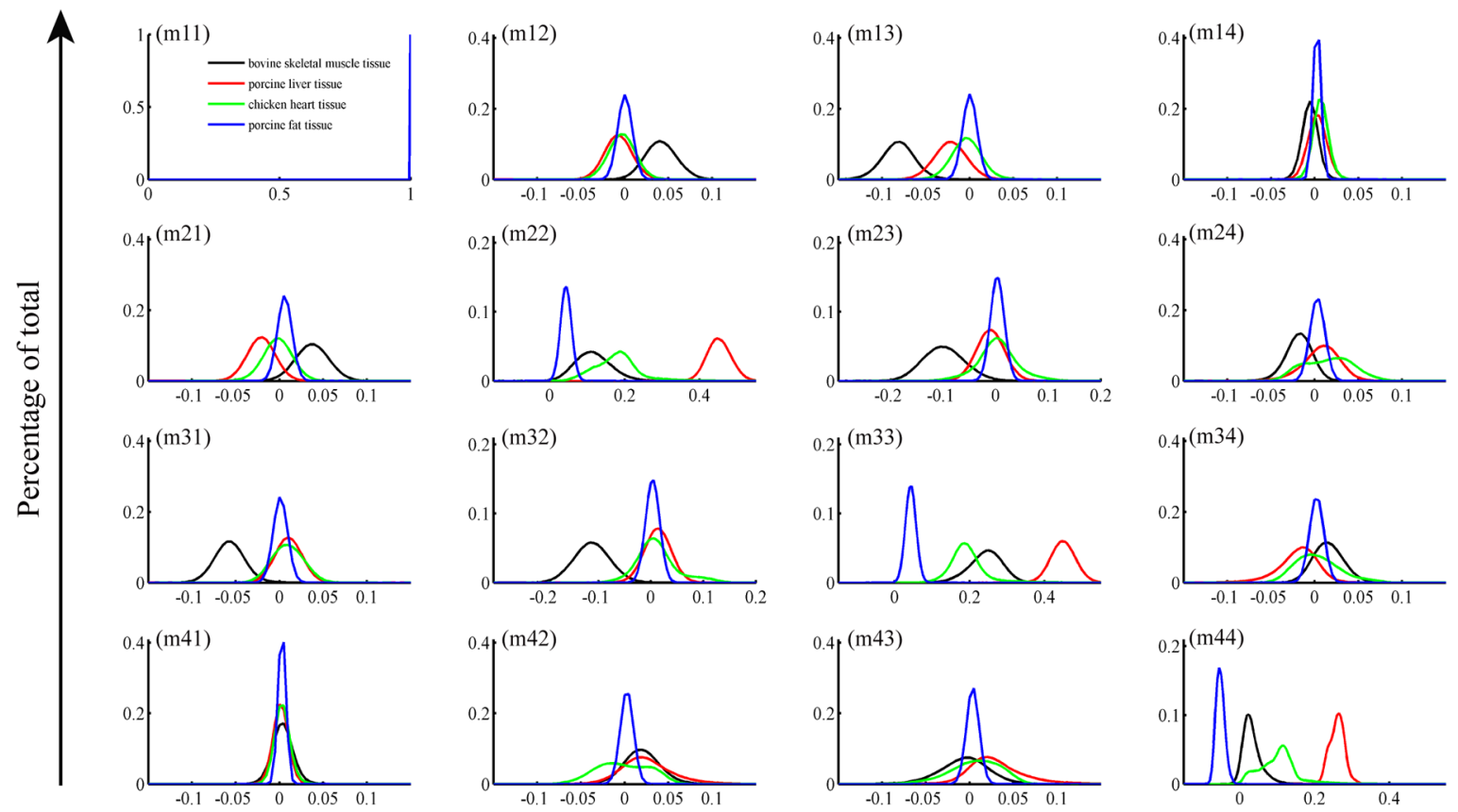

Values of Mueller matrix elements

Fig. 6 Frequency distribution histogram (FDH) of Mueller matrix elements of different tissue samples: bovine skeletal muscle (black lines), porcine liver (red lines), chicken heart (green lines), and porcine fat (blue lines). The areas under the curves are normalized to 1 , and the horizontal axis is divided into 400 parts. 
Table 3 Central moment parameters of the Mueller matrix elements for different tissues. ${ }^{a}$

\begin{tabular}{|c|c|c|c|c|c|c|c|c|c|c|c|c|c|c|c|}
\hline & $\mathrm{m} 12$ & $\mathrm{~m} 13$ & $\mathrm{~m} 14$ & $\mathrm{~m} 21$ & m22 & m23 & m24 & m31 & m32 & m33 & m34 & $\mathrm{m} 41$ & $\mathrm{~m} 42$ & $\mathrm{~m} 43$ & $\mathrm{~m} 44$ \\
\hline $\mathrm{M} / \mathrm{P} 1$ & 0.039 & -0.084 & -0.008 & 0.035 & 0.114 & -0.102 & -0.021 & -0.060 & -0.115 & 0.241 & 0.013 & 0.001 & 0.017 & -0.009 & 0.029 \\
\hline L/P1 & -0.010 & -0.024 & 0.000 & -0.023 & 0.449 & -0.013 & 0.005 & 0.008 & 0.010 & 0.447 & -0.021 & -0.001 & 0.022 & 0.027 & 0.257 \\
\hline $\mathrm{H} / \mathrm{P} 1$ & -0.006 & -0.006 & 0.004 & -0.004 & 0.176 & 0.000 & 0.013 & 0.007 & 0.008 & 0.190 & 0.001 & 0.001 & -0.004 & 0.004 & 0.100 \\
\hline F/P1 & -0.002 & -0.002 & 0.000 & 0.003 & 0.041 & 0.000 & 0.001 & -0.001 & 0.001 & 0.041 & 0.000 & 0.000 & 0.000 & 0.001 & -0.055 \\
\hline M/P2 & 0.016 & 0.017 & 0.008 & 0.017 & 0.042 & 0.035 & 0.013 & 0.015 & 0.030 & 0.039 & 0.015 & 0.010 & 0.018 & 0.025 & 0.021 \\
\hline L/P2 & 0.014 & 0.017 & 0.009 & 0.014 & 0.028 & 0.024 & 0.018 & 0.014 & 0.022 & 0.029 & 0.019 & 0.008 & 0.025 & 0.027 & 0.017 \\
\hline $\mathrm{H} / \mathrm{P} 2$ & 0.014 & 0.015 & 0.008 & 0.015 & 0.051 & 0.033 & 0.026 & 0.016 & 0.034 & 0.038 & 0.023 & 0.008 & 0.027 & 0.026 & 0.045 \\
\hline F/P2 & 0.007 & 0.007 & 0.004 & 0.007 & 0.013 & 0.012 & 0.007 & 0.007 & 0.012 & 0.012 & 0.007 & 0.004 & 0.006 & 0.006 & 0.010 \\
\hline M/P3 & 0.071 & -0.016 & -0.016 & -0.002 & 0.230 & 0.103 & -0.141 & 0.050 & 0.037 & -0.215 & 0.141 & 0.064 & 0.055 & -0.178 & 0.824 \\
\hline L/P3 & 0.040 & -0.004 & 0.030 & 0.003 & 0.073 & 0.004 & -0.250 & 0.006 & -0.020 & 0.028 & -0.447 & 0.000 & 0.360 & 0.496 & -0.101 \\
\hline $\mathrm{H} / \mathrm{P} 3$ & 0.016 & 0.000 & 0.058 & -0.030 & 0.382 & 0.032 & -0.038 & 0.051 & 0.614 & 0.844 & 0.380 & 0.125 & 0.008 & -0.289 & 0.491 \\
\hline F/P3 & 0.006 & -0.005 & -0.003 & -0.004 & 0.037 & 0.003 & 0.009 & 0.013 & -0.003 & 0.025 & -0.009 & 0.005 & -0.006 & 0.027 & 0.205 \\
\hline $\mathrm{M} / \mathrm{P} 4$ & 2.372 & 2.509 & 2.445 & 2.359 & 2.446 & 2.357 & 2.444 & 2.426 & 2.367 & 2.470 & 2.386 & 2.495 & 2.492 & 2.527 & 3.388 \\
\hline L/P4 & 2.374 & 2.408 & 2.437 & 2.369 & 2.364 & 2.357 & 2.537 & 2.362 & 2.373 & 2.376 & 2.804 & 2.400 & 2.696 & 3.040 & 2.312 \\
\hline $\mathrm{H} / \mathrm{P} 4$ & 2.401 & 2.493 & 2.557 & 2.411 & 3.338 & 2.852 & 2.061 & 2.296 & 3.295 & 4.336 & 2.557 & 2.539 & 2.024 & 2.402 & 3.818 \\
\hline F/P4 & 2.351 & 2.358 & 2.375 & 2.349 & 2.361 & 2.359 & 2.353 & 2.354 & 2.356 & 2.358 & 2.374 & 2.359 & 2.360 & 2.369 & 2.368 \\
\hline
\end{tabular}

${ }^{\mathrm{a}} \mathrm{M}, \mathrm{L}, \mathrm{H}$, and $\mathrm{F}$ represent the bovine skeletal muscle, porcine liver, chicken heart, and porcine fat, respectively.

moment parameters are calculated and listed in Table 3. In the discussions above, we have summarized the characteristic features of anisotropic and isotropic tissues using Figs. 4, 5 and Tables 1, 2. Here, we can confirm the relations between the microstructures and the distributions of Mueller matrix elements from Fig. 6 and Table 3 more clearly.

First, the anisotropic and isotropic tissues can be distinguished by using the diagonal elements. The porcine liver (red lines) and fat (blue lines) tissues are predominantly isotropic; therefore, their $\mathrm{m} 22$ and $\mathrm{m} 33$ curves are almost the same (for example, for the fat tissue, P1 of the $\mathrm{m} 22$ and $\mathrm{m} 33$ are 0.041 and $0.041, \mathrm{P} 2$ are 0.013 and 0.012 ). The anisotropic bovine skeletal muscle (black lines) and chicken heart (green lines) tissues, however, display differences between the m22 and $\mathrm{m} 33$, which become more prominent as the anisotropy increases. Table 3 shows that the differences in P1 of $\mathrm{m} 22$ and m33 elements for anisotropic skeletal muscle and heart samples are 0.127 and 0.014 , respectively. This is because that the fibers in skeletal muscle sample are well aligned in almost the same direction, while in heart sample the fibers are distributed in different orientations. For isotropic fat and liver tissues, the differences in $\mathrm{P} 1$ of $\mathrm{m} 22$ and $\mathrm{m} 33$ elements are 0 and 0.002 . This is because the fat tissue is totally isotropic, while the liver sample has a small portion of birefringent connective tissues. Second, we also notice that the distribution widths of the FDHs (the values of P2) for bovine skeletal muscle, chicken heart, and porcine liver samples are larger than the fat sample, indicating more complicated microstructures for these metabolic exuberant tissues. The FDHs of the $\mathrm{m} 24, \mathrm{~m} 42, \mathrm{~m} 34$, and $\mathrm{m} 43$ elements for skeletal muscle, heart, and liver tissues show small positive or negative values, which are related to the birefringent structures in these tissues. The signs of the elements can be used to determine the aligned fibers directions. ${ }^{18}$ At last, the different depolarization power of tissues can also be observed from Fig. 6 and Table 3: the liver tissue sample has the largest P1 values of the diagonal elements, showing the smallest depolarization power, while the smallest P1 values of the diagonal elements indicate the most prominent depolarization property of the fat tissue. ${ }^{10}$ Although more studies are still needed to reveal the relationships between the derived parameters and tissue morphology, it has been shown that the parameter P2 should be sensitive to the complexity of a sample: a large value of P2 means that the measured polarization data are distributed in a wider range, indicating a complex structural feature of the tissue. The parameter P3 should be sensitive to the heterogeneity of a sample: a large value of P3 means that the measured polarization data are unequally distributed around the expected value. The parameter $\mathrm{P} 4$ can also be used to reflect the complexity of a sample: a large $\mathrm{P} 4$ shows that most measured polarization data are distributed very close to the mean value, meaning that the microstructural features are similar.

In summary, from the results discussed above, we can conclude that: (1) the shapes of FDHs (values of P2, P3, and P4) are orientation insensitive, therefore can reflect some intrinsic structural properties of the samples. (2) The FDHs and corresponding central moment parameters of Mueller matrix elements are good quantitative indicators of the microstructures. Although the 2-D images contain more detailed structural information 

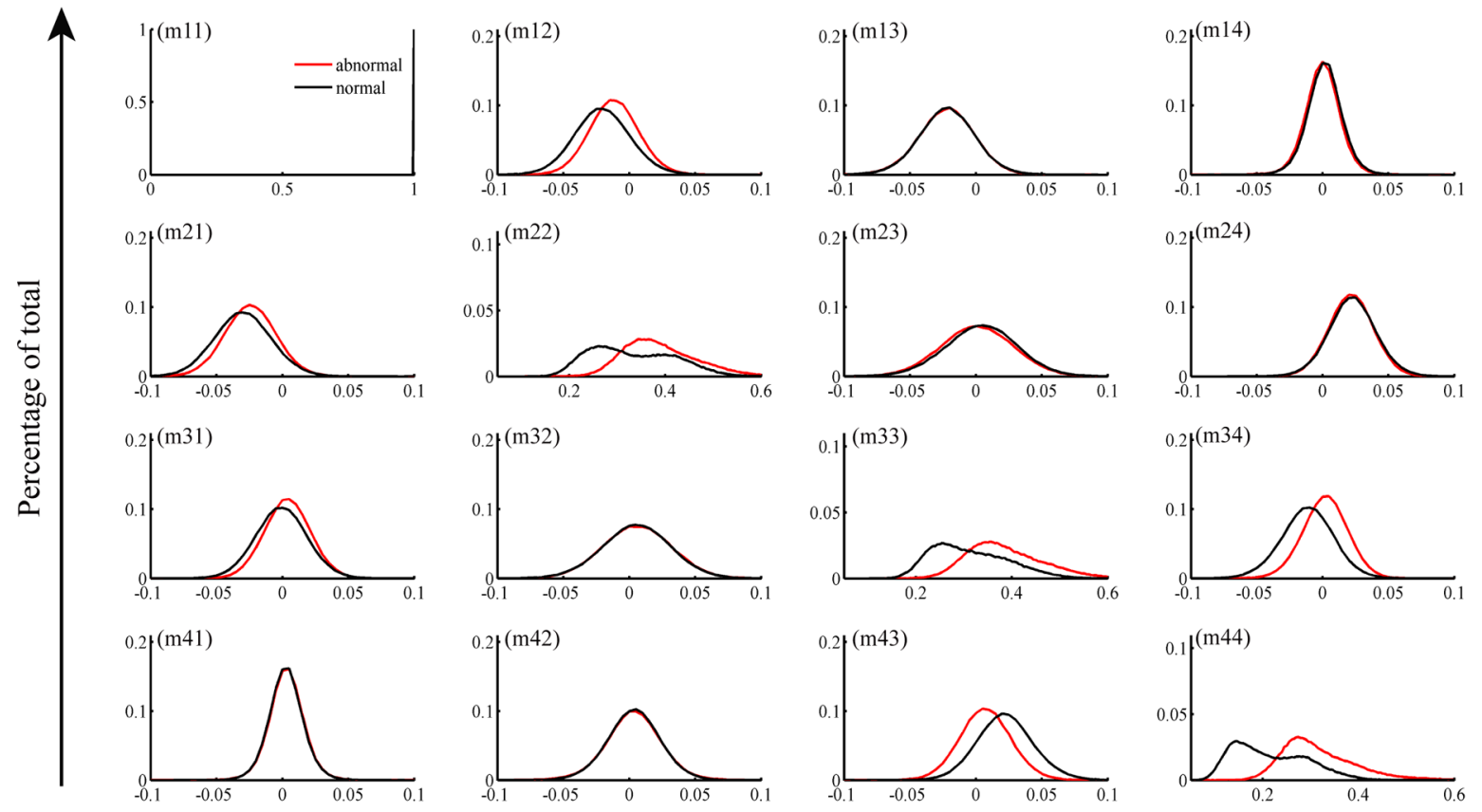

Values of Mueller matrix elements

Fig. 7 Frequency distribution histogram (FDH) of Mueller matrix elements of human cervical cancer tissue: normal region (black lines) and abnormal region (red lines). The areas under the curves are normalized to 1 , and the horizontal axis is divided into 400 parts.

as described in our previous studies, transforming the images into FDHs and quantitative central moment parameters can reveal the dominant features of tissues.

\subsection{Application to Human Cervical Cancerous Tissues}

To testify the potential applications of the pixel FDHs and central moment parameters on diagnosis, we take the Mueller matrices of an unstained $28-\mu \mathrm{m}$-thick slice of human cervical cancerous tissue prepared and provided by the Shenzhen Sixth People's (Nanshan) Hospital (ID: 120900924), the detailed information of the tissue can be found in Ref. 10. This work was approved by the Ethics Committee of the
Shenzhen Sixth People's (Nanshan) Hospital. We also choose $700 \times 700$ squares from both the normal and abnormal regions of the 2-D images, and then calculate the FDHs and central moments of Mueller matrix elements shown as shown in Fig. 7 and Table 4.

It can be observed from Fig. 7 and Table 4 that the normal and abnormal cervical tissues represent different structural features. The normal region has larger anisotropy (more prominent difference between the $\mathrm{m} 22$ and $\mathrm{m} 33$ elements) and depolarization properties (smaller values of the $\mathrm{m} 22, \mathrm{~m} 33$, and $\mathrm{m} 44$ elements) than the abnormal region. The positive and negative values of the $\mathrm{m} 34$ and $\mathrm{m} 43$ elements also indicate the existence of birefringence in normal tissue (P1 of the m34 and $\mathrm{m} 43$ are -0.014 and 0.020 ), while for abnormal tissue, the birefringent

Table 4 Central moment parameters of the Mueller matrix elements for human cervical cancerous tissue.

\begin{tabular}{|c|c|c|c|c|c|c|c|c|c|c|c|c|c|c|c|}
\hline & $\mathrm{m} 12$ & $\mathrm{~m} 13$ & $\mathrm{~m} 14$ & m21 & m22 & $\mathrm{m} 23$ & m24 & m31 & m32 & m33 & m34 & m41 & $\mathrm{m} 42$ & m43 & $\mathrm{m} 44$ \\
\hline abnormal/P1 & .014 & .025 & .003 & .027 & 0.386 & 02 & 0.019 & 0.001 & 0.003 & 0.383 & 01 & 0.001 & 0.001 & .005 & 0.312 \\
\hline normal/P1 & -0.023 & -0.024 & 0.001 & -0.032 & 0.328 & 0.001 & 0.020 & -0.003 & 0.003 & 0.308 & 14 & 0.000 & 0.001 & 0.020 & .212 \\
\hline abnorm & 0.016 & 0.018 & 0.011 & 0.017 & 0.068 & 0.024 & 0.015 & 0.015 & 0.023 & 0.070 & 0.015 & 0.011 & 0.018 & 0.017 & 0.066 \\
\hline normal/P2 & 0.018 & 0.018 & 0.011 & 0.019 & 0.080 & 0.024 & 0.016 & 0.017 & 0.023 & 0.071 & 0.017 & 0.011 & 0.017 & 0.019 & 0.070 \\
\hline abnormal/P3 & 0.034 & -0.057 & -0.009 & 0.003 & 0.479 & -0.0 & -0.0 & 0.008 & -0.009 & 0.458 & -0.0 & 0.005 & -0 & 0.033 & 0.664 \\
\hline normal/P3 & 0.050 & -0.029 & -0.011 & 0.025 & 0.249 & -0.056 & 0.020 & 0.006 & -0.030 & 0.382 & -0.061 & 0.035 & -0.034 & 0.081 & 0.370 \\
\hline abnormal/P4 & 2.387 & 2.410 & 2.520 & 2.380 & 2.555 & 2.356 & 2.371 & 2.374 & 2.358 & 2.514 & 2.402 & 2.539 & 2.516 & 2.403 & 2.845 \\
\hline normal/P4 & 2.395 & 2.423 & 2.516 & 2.378 & 1.975 & 2.394 & 2.441 & 2.390 & 2.391 & 2.211 & 2.421 & 2.522 & 2.435 & 2.434 & 2.043 \\
\hline
\end{tabular}


effect becomes limited. ${ }^{9-10}$ Besides, we also notice that the values of $\mathrm{P} 3$ and $\mathrm{P} 4$ for normal and abnormal tissues represent large difference in some elements, such as the $\mathrm{m} 22, \mathrm{~m} 33$, and $\mathrm{m} 44$. These preliminary studies show that the FDHs and central moment parameters may have the potential to give quantitative or semiquantitative criteria for cancerous tissues detections.

\section{Conclusion}

In this work, we take the backscattering Mueller matrices of tissues of distinctively different microstructures: bovine skeletal muscle tissue, porcine liver tissue, chicken heart tissue, and porcine fat tissue, then use the pixel FDH and central moment analysis to transform the 2-D Mueller matrix images to a group of quantitative indicators for characterizing the dominant structural properties of tissues. By rotating anisotropic skeletal muscle sample and isotropic liver tissue, we find that the central moment parameters $\mathrm{P} 2, \mathrm{P} 3$, and $\mathrm{P} 4$ are insensitive to sample orientation directions. Comparisons among different tissues testify that the distribution behavior and corresponding central moment parameters of Mueller matrix elements are good indicators of the microstructures of tissues. A preliminary application to human cervical cancerous tissues shows that the distribution curves and central moment parameters may have the potential to give quantitative or semiquantitative criteria for cancerous tissues detections.

\section{Acknowledgments}

This work has been supported by the National Natural Science Foundation of China (NSFC) under Grant Nos. 11174178, 11374179, 61205199, 61405102, and the Science and Technology Project of Shenzhen Grant Nos. CXZZ20140509172959978 and JCY20130402145002404.

\section{References}

1. R. S. Gurjar et al., "Imaging human epithelial properties with polarized light-scattering spectroscopy," Nat. Med. 7(11), 1245-1248 (2001).

2. B. Kunnen et al., "Application of circularly polarized light for noninvasive diagnosis of cancerous tissues and turbid tissue-like scattering media," J. Biophotonics 8(4), 317-323 (2015).

3. N. Ghosh and I. A. Vitkin, "Tissue polarimetry: concepts, challenges, applications, and outlook," J. Biomed. Opt. 16(11), 110801 (2011).

4. S. L. Jacques, J. C. Ramella-Roman, and K. Lee, "Imaging skin pathology with polarized light," J. Biomed. Opt. 7(3), 329-340 (2002).

5. R. R. Anderson, "Polarized light examination and photography of the skin," Arch. Dermatol. 127(7), 1000-1005 (1991).

6. S. Alali and I. A. Vitkin, "Polarized light imaging in biomedicine: emerging Mueller matrix methodologies for bulk tissue assessment," J. Biomed. Opt. 20(6), 061104 (2015).

7. E. Du et al., "Mueller matrix polarimetry for differentiating characteristic features of cancerous tissues," J. Biomed. Opt. 19(7), 076013 (2014).
8. P. Shukla and A. Pradhan, "Mueller decomposition images for cervical tissue: Potential for discriminating normal and dysplastic states," Opt. Express 17(3), 1600-1609 (2009).

9. A. Pierangelo et al., "Polarimetric imaging of uterine cervix: a case study," Opt. Express 21(12), 14120-14130 (2013).

10. M. Sun et al., "Characterizing the microstructures of biological tissues using Mueller matrix and transformed polarization parameters," Biomed. Opt. Express 5(12), 4223-4234 (2014).

11. A. Pierangelo et al., "Multispectral Mueller polarimetric imaging detecting residual cancer and cancer regression after neoadjuvant treatment for colorectal carcinomas," J. Biomed. Opt. 18(4), 046014 (2013).

12. A. Pierangelo et al., "Ex vivo photometric and polarimetric multilayer characterization of human healthy colon by multispectral Mueller imaging," J. Biomed. Opt. 17(6), 066009 (2012).

13. A. Pierangelo et al., "Ex-vivo characterization of human colon cancer by Mueller polarimetric imaging," Opt. Express 19(2), 1582-1593 (2011).

14. M. Dubreuil et al., "Mueller matrix polarimetry for improved liver fibrosis diagnosis," Opt. Lett. 37(6), 1061-1063 (2012).

15. W. Wang et al., "Roles of linear and circular polarization properties and effect of wavelength choice on differentiation between ex vivo normal and cancerous gastric samples," J. Biomed. Opt. 19(4), 046020 (2014).

16. J. Jagtap et al., "Quantitative Mueller matrix fluorescence spectroscopy for precancer detection," Opt. Lett. 39(2), 243-246 (2014).

17. I. Ahmad et al., "Ex vivo characterization of normal and adenocarcinoma colon samples by Mueller matrix polarimetry," J. Biomed. Opt. 20(5), 056012 (2015).

18. H. He et al., "Mapping local orientation of aligned fibrous scatterers for cancerous tissues using backscattering Mueller matrix imaging," $J$. Biomed. Opt. 19(10), 106007 (2014).

19. M. Sun et al., "Probing microstructural information of anisotropic scattering media using rotation-independent polarization parameters," Appl. Opt. 53(14), 2949-2955 (2014).

20. H. He et al., "Two-dimensional and surface backscattering Mueller matrices of anisotropic sphere-cylinder scattering media: a quantitative study of influence from fibrous scatterers," J. Biomed. Opt. 18(4), 046002 (2013).

21. R. M. A. Azzam, "Photopolarimetric measurement of the Mueller matrix by Fourier analysis of a single detected signal," Opt. Lett. 2(6), 148-150 (1978).

22. D. H. Goldstein, "Mueller matrix dual-rotating retarder polarimeter," Appl. Opt. 31(31), 6676-6683 (1992).

23. D. H. Goldstein and R. A. Chipman, "Error analysis of a Mueller matrix polarimeter," J. Opt. Soc. Am. A 7(4), 693-700 (1990).

24. D. B. Chenuault, J. L. Pezzaniti, and R. A. Chipman, "Mueller matrix algorithms," Proc. SPIE 1746, 231-246 (1992).

25. R. Liao et al., "Rotating linear polarization imaging for quantitative characterization of anisotropic tissues," J. Biomed. Opt. 15(3), 036014 (2010).

26. G. Grimmett and D. Stirzaker, Probability and Random Processes, Oxford University Press, New York (2001).

27. X. D. Li, "Value at risk based on the volatility, skewness and kurtosis," Working Paper, RiskMetrics Group (1999).

28. V. A. Ushenko, O. V. Dubolazov, and A. O. Karachevtsev, "Two wavelength Mueller matrix reconstruction of blood plasma films polycrystalline structure in diagnostics of breast cancer," Appl. Opt. 53(10), B128-B139 (2014).

Biographies for the authors are not available. 